\title{
Mythologie und Kosmetik
}

\author{
Mythology and Cosmetics
}

\section{Bibliografie}

DOI http://dx.doi.org/

10.1055/s-0033-1344888

Akt Dermatol 2013; 39: 476-481

(c) Georg Thieme Verlag KG

Stuttgart · New York

ISSN 0340-2541

Korrespondenzadresse

Prof. Dr. med. Ernst G. Jung

Maulbeerweg 20

69120 Heidelberg

ernst.g.jung@t-online.de

\section{Zusammenfassung \\ $\nabla$}

Kosmetik ist tief in der Mythologie verankert und zumeist von Eros, der „begehrlichen Liebe“, getrieben. Dieser ist allgegenwärtig in unserem Bewusstsein und im Unbewussten, spricht durch Zeichen, Bilder und Symbole, lebt in Riten und Konventionen und durchzieht gewaltig die kulturelle Entwicklung; den „Gang des Geistes“ genauso wie den „Ruf des Fleisches“.

Kultur als Bereicherung und Ergänzung zur Natur wurde den Menschen einst von den Göttern direkt oder durch „götterähnliche Kulturbringer“ vermittelt. Dies geschah in der vorhistorischen Zeit und lebt in Mythen und Riten weiter. In allen Kulturen sind solche, fast einheitlich zu nennende Vorgänge tradiert, sodass man vom kulturellem Gedächtnis [1], von archetypischer Verhaltensweise [2] oder, in Anlehnung an die Gene, von Memen [3] spricht.

Das Christentum hat diese frühen Bilder recht konsequent übernommen und seinen Heilsgeschichten einverleibt. Solches ist exemplarisch in der 11. Vision des Salemer Codex III von Hildegard von Bingen dargestellt ( $\bullet$ Abb. 1).

Sowohl in der Erinnerung als auch in Bräuchen und Ritualen treten diese verblüffend ähnlichen mythischen Figuren immer wieder auf. Dargestellt werden sie regelmäßig durch anthropomorphe Masken mit tierischen Merkmalen. Respekt und Furcht sollen sie einflößen, weshalb sie mit den typischen Attributen der Hässlichkeit ausgestattet sind [4-7].

Auch in Kinderzeichnungen [8,9] kommen Masken oder maskenartige Gesichter gelegentlich zur Darstellung. Traumbilder oder persönliche Erfahrungen mögen dem zugrunde liegen. Bizarre Betonungen der Augenhorizontalen und exzessive, schreckliche Verzerrungen der Mundhorizontalen sind charakteristisch, wie sie bei der
Es beginnt mit der Gesichtserkennung und der Spanne zwischen Schönheit und Hässlichkeit. Kosmetik will, ausgehend von der Schönheit, die sexuelle Attraktivität steigern. Die Betonung der Augenhorizontalen und besonders die Variation von Form und Farbe der Mundpartie sind die effektiven Elemente dazu. Eine Überzeichnung provoziert unvermittelt Hässlichkeit, was am Beispiel von Masken gezeigt wird. Davor wird gewarnt.

Darstellung mythischen oder traditionellen Maskentreibens vorherrschen $[7,13]$.

Besondere Beachtung wurde den Zeichnungen und Gemälden von bildnerisch begabten Personen mit Down-Syndrom zugemessen. Umfangreiche Darstellungen der Werke von Jane Cameron und von Willibald Lassenberger [9] zeigen nur gelegentlich Bilder mit Masken oder maskenartigen Gesichtern ( $\bullet$ Abb.2). Auch hier werden die Gesichtshorizontalen bizarr vergrößert und schrecklich verformt. Tierische Attribute kommen zuweilen hinzu, ähnlich wie bei den rituellen und mythischen Masken.

Auch bei Patienten mit psychischen Erkrankungen wurde nach dem Phänomen Masken und deren bildhafter Deutung mit assoziativen Verfahren gesucht. Der Rorschachtest ist eine Methode der Psychodiagnostik [10], benannt nach dem schweizerischen Psychiater Hermann Rorschach (1884-1922), und beruht auf der Formdeutung von Tintenklecksbildern. Die Bedeutung von Masken in diesem Testverfahren ist vom Psychiater Ronald Kuhn (1912-2005) 1954 erstmals in Buchform [11,12] herausgearbeitet worden. Von 1042 Patienten der psychiatrischen Klinik in Münsterlingen (Thurgau/CH) wurden 912 einoder mehrmals einem Rorschachtest unterzogen. Masken wurden von 88 dieser Patienten (9,6\%) mehr oder weniger präzise beschrieben. 


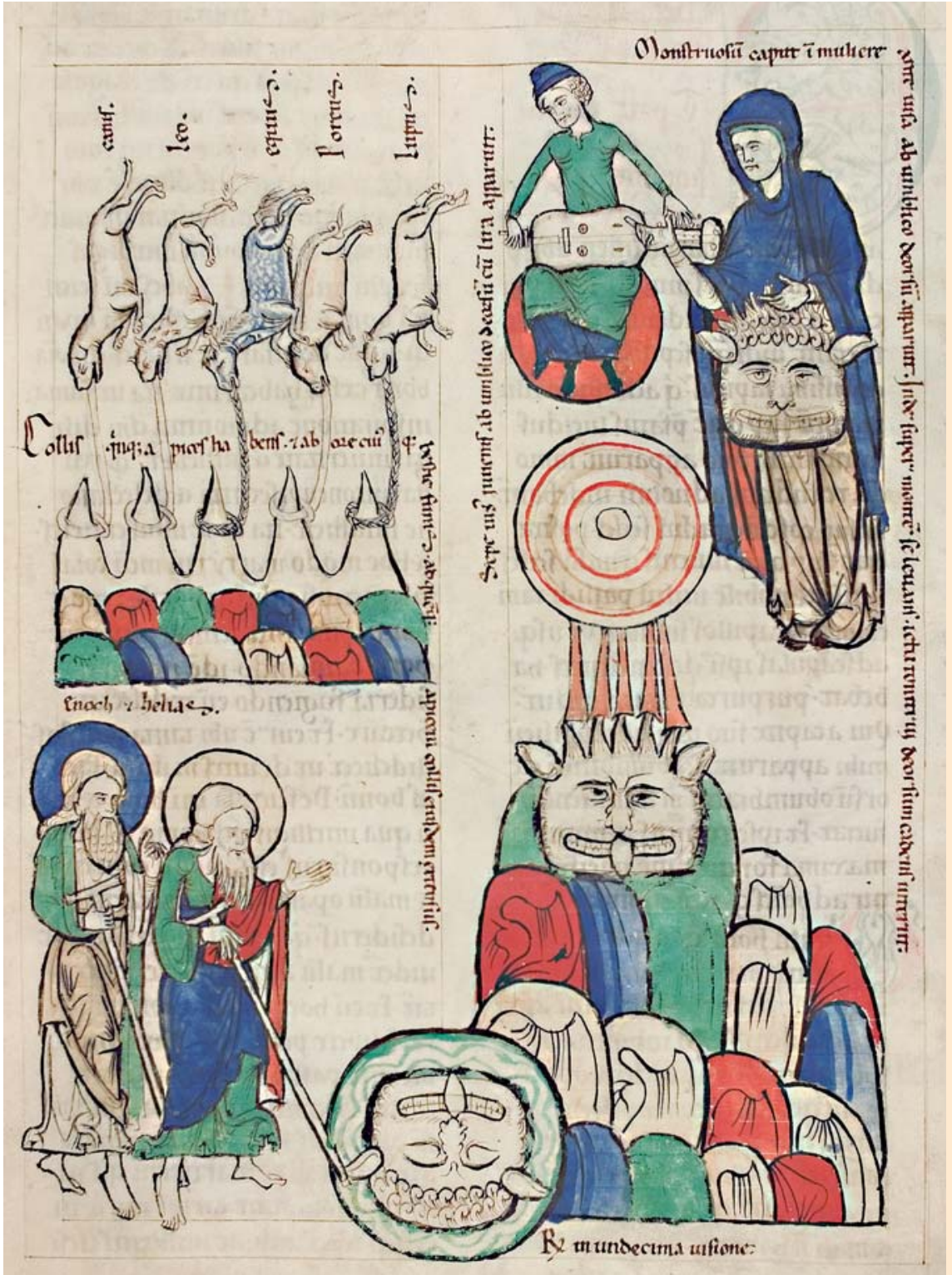

Abb.1 Hildegard von Bingen: Liber Scivias III, 11; Rechts oben die blaue Figur als Allegorie der Kirche ist beschäftigt mit dem „Haupt des Bösen, mit eselsgleichen Ohren und mit Nase und Rachen wie ein Löwe“", welches in der Bildmitte anmaßend sich erhebt und endlich bewältigt, abgestürzt und tot (verkehrt) am unteren Bildrand liegt (Bild: UB HD: Cod. Sal. X,16: fol. 177r).

Vorwiegend sind es Menschenmasken, gelegentlich mit Tierattributen. Die Bilderkennung $[7,13]$ erfolgte anhand der Augenhorizontalen und der besonders pointiert bezeichneten Gesichtsvertikalen mit Nasenbetonung. Charakterisiert werden die Masken oft als Clowns, Gaukler, Fastnachtsfiguren und seltener als Teufel, Gasmaske oder Totenmaske. Betont wird regelmäßig das Groteske, das Grimmige, das Hässliche, sowie Karikatur und Verfremdung. Ästhetik als Schönheit und Attraktivität wird kaum angeführt.

\section{Das kulturelle Gedächtnis hat unterschiedliche Tiefen $\nabla$}

Wir tragen alle unsere Vergangenheiten in uns, die eigenen und diejenigen unserer Vorfahren. Aus Masken und Bildern spricht der Ursprung, wirkt mit Schrecken und fordert Furcht, Gottesfurcht zunächst. Archaische Ängste wirken fort, und ebenso die Mühen des Bestehens der Natur und der Mitmenschen. Mythen erinnern an die Wege der stufenweisen Sozialisation. Alles wirkt zusammen, alles ist präsent, in jedem von uns, aber auch in den Mythen und Märchen, den Träumen und in mannigfachen Sym- bolen, die uns begleiten. Gemeinsam ist vieles, weshalb Erklärung gesucht und Benennung gegeben wird. Kulturelles Gedächtnis [1], kollektives Unbewusstsein [2] oder ein System von Memen („memory genes“, [3]) sind die Kennworte. Geschehnisse werden reflektiert, die mehrere zehntausend Jahre zurückliegen, in der vorhistorischen, der mythischen Epoche.

Im Gegensatz zum Kunstverständnis, in welchem die Hässlichkeit als Antiposition zur Schönheit begriffen wird [5,14], spielt bei den Maskensymbolen die Hässlichkeit die traditionelle, in den Mythen verankerte Leitrolle. Und die Schönheit wird, wenn überhaupt, als Gegensatz zur Hässlichkeit der mythischen Symbole verstanden.

Schönheit ist in der Kunst lange Zeit nach dem Maß der Natur bestimmt worden und ist dennoch ein junges Ideal, das sich nicht auf die Urzeiten rückverfolgen lässt. Es hat sich in den frühen Hochkulturen, wohl sieben- bis neuntausend Jahre vor unserer Zeitrechnung, entwickelt. In Stein gehauen wird es reichlich dokumentiert und hat sich rasant als ein bemerkenswertes und erstrebenskräftiges Ziel herausgebildet. Damit ist Schönheitspflege, Kosmetik, als Instrument der Zielführung aufgekommen und hat sich in Wellen durch die Jahrtausende [15] bis heute fort- 


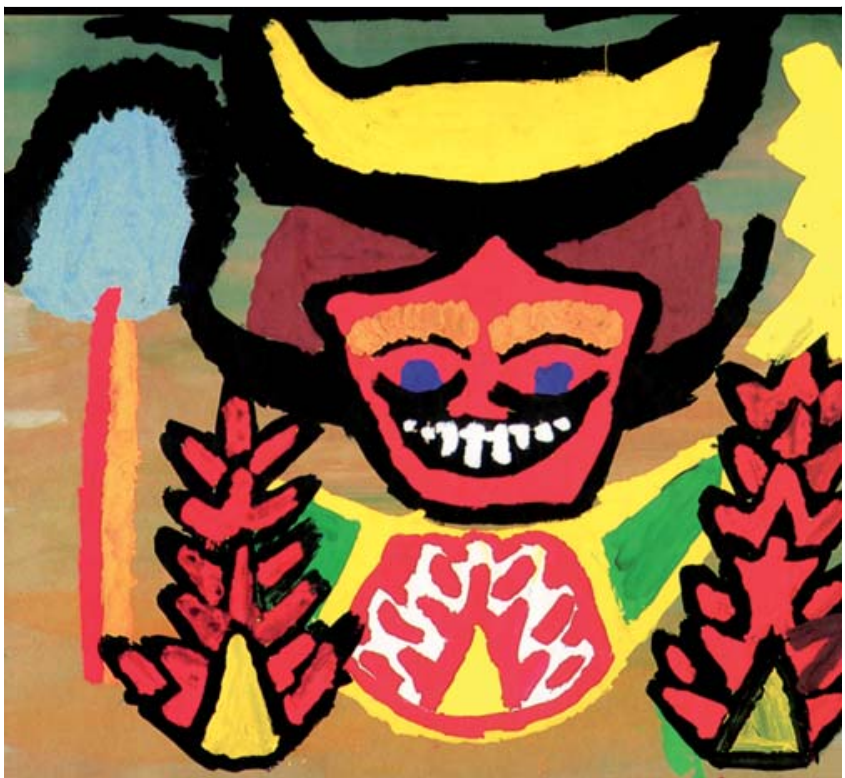

Abb. 2 Krampus von Willibald Lassenberger [9]. Krampus ist eine Figur mit einer hässlich-schrecklichen Menschenmaske und tierischen Attributen, Begleiter von St. Nikolaus in den Adventsbräuchen der Ostalpen.

entwickelt. Schönheit wird als soziale Macht verstanden und verbindet die Vorstellung, respektive den Drang nach Gesundheit, Jugendlichkeit und sexueller Attraktivität. Argumentiert wird dies mit einer Lust zur Selbstdarstellung und ermöglicht eine Umgestaltung der Partnerwahl. Letztere war traditionell ausgerichtet auf die Lebensaufgaben der Gemeinschaften, Familie, Sippe und Volk, wobei dem Manne die Ernährung und die Sicherheit oblag, während die Frau für die Aufzucht der Kinder garantierte. Daran orientierte sich die Partnerwahl. Im Laufe der kulturellen Entwicklung mit tragfähigen Sozialstrukturen, mit medizinischem Fortschritt, mit Freiheiten aller Arten und nicht zuletzt durch eine effiziente Geburtenkontrolle ist Partnerwahl zu einem wiederholten, ja permanenten Anliegen geworden [7]. Dazu bedarf es zweier besonderer Qualitäten. Das eine ist die Selbstsicherheit, die mit der Selbstdarstellung verknüpft ist und oft Elemente von Selbstinszenierung und Körpermodifizierung trägt. Das andere Element ist die sexuelle Attraktivität, die durch Modifizierung gesteigert und damit regelrecht inszeniert wird.

\section{Die sexuelle Attraktivität als neue Qualität $\nabla$}

Seit dem klassischen Altertum spielt die sexuelle Attraktivität, zunächst als Teilqualität der Schönheit, dann immer mächtiger eine eigene, bedeutende Rolle. Dabei spielen des menschliche Gesicht und seine Ausdrucksformen die primäre und meist maßgebliche Rolle. Dies gilt es näher zu beleuchten.

Die sexuelle Attraktivität eines Menschengesichtes ist zwischen Hässlichkeit auf der einen Seite einer virtuellen Skala und der Schönheit auf der anderen anzusiedeln.

Die Hässlichkeit ist in natura zwar vorhanden, in ihrer besonders eindrücklichen und stereotypen Ausprägung aber ist sie in unserem Unbewussten gegenwärtig und wirksam. In den entsprechenden Masken der mythischen Figuren ist Hässlichkeit noch immer und in allen Kulturbereichen präsent und wird tradiert. Hässlichkeit ist aber erst nachdem Karl Rosenkranz 1853 [4] die
„Ästhetik der Hässlichkeit“ formulierte zum Gegenstand von kulturellen und anthropologischen Studien geworden. Hässlichkeit und Schönheit werden seither mit derselben Akribie, Ernsthaftigkeit und Phantasie bearbeitet. Instrument und Engagement sind also gleich, als Gegenstand aber sind sie auf einer affektiven Skala diametral entgegengesetzt, ein richtiges Gegensatzpaar. Damit ist das eine Ende der virtuellen Skala, die Hässlichkeit nämlich, mythologisch fest verankert und allgemein anerkannt. Das andere Ende bestimmt die auch recht allgemein anerkannte Schönheit, die seit dem klassischen Altertum immer wieder, intensiv und phantasievoll beschrieben, gepriesen und dokumentiert ist. Sie ist, gegenüber der Hässlichkeit, ein viel jüngeres Element unseres Kulturgutes. Sie hat eine beträchtliche Spielbreite und unterliegt ethnischen Verschiedenheiten. Es ist aber nie gelungen, sie mit Mustergliederungen oder mathematischen Formulierungen zu fassen.

\section{Von Schönheit und Attraktivität \\ $\nabla$}

Idealtypische Schönheit wirkt distanziert und es mangelt ihr an Attraktivität. Denn es gibt keine Schönheit ohne individuelle Merkmale, die als Blickfang den Betrachter „auf einen Blick“, in der Gegenwart von knapp 3 Sekunden anzulocken und zu fesseln vermögen [13]. Als Blickfang dienen einerseits die Augen mit ihren speziellen, auch rassischen Eigenheiten, „Vom Schlitzauge bis zum Mandelauge“; eben das, was in der unmittelbaren Umwelt als besonders auffällt. Dem kann chirurgisch oder kosmetisch nachgeholfen werden durch Veränderung von Form und Ausrichtung. Häufig wird mit den Brauen und Wimpern gespielt. Auch die Nase wird oft modifiziert. Breite Nasenflügen, Buckel oder Kerben am Nasenrücken werden operativ bleibend verbessert und dem Vorbild der „römischen Nase“ angenähert, wobei die feine Nase direkt aus der Stirne fast geradlinig entspringen soll. Eine besondere Bedeutung liegt bei Mund und Lippen. Zentral im Gesicht stellen diese optischen Einheiten den primären und nachhaltigsten Blickpunkt dar, der uns von der allgemeinen Attraktivität direkt zur sexuellen Attraktivität weiterführt.

Fakt ist also, dass die „ideale Schönheit“ keineswegs identisch ist mit Attraktivität und schon gar nicht mit deren sexueller Variante. Schönheit impliziert nicht nur „Gefallen“, sondern auch „edle Gesinnung“ und soziales Vorbild. Zuweilen vermittelt sie auch Distanz, Respekt und Berührungsängste. Ganz anders die sexuelle Attraktivität, sie ist egoistisch, rücksichtslos und zielorientiert. Dies wird erreicht durch Hinzuziehung und Steigerung der Blickpunkte, verstärkt und zuweilen bizarr verzerrt durch besondere Elemente, die wir aus der Darstellung der Hässlichkeit schon kennen. Sie fallen auf, fangen den Blick und bringen die erogenen Zonen aufdringlich in den Vordergrund. Solche Elemente werden dem schönen Grundmuster übergestülpt, wobei Ausmaß und Verteilung variieren und erst durch die Effekte in der Anwendung bestätigt, verworfen oder modifiziert werden. Das ist das moderne Feld der Kosmetik. Und es wird weltweit intensiv und vielfältig begangen.

\section{Die Maske des Eros \\ $\nabla$}

„Das Schöne ist nichts als des Schrecklichen Anfang, das wir gerade noch ertragen“, so schreibt R. M. Rilke in seiner „1. Duineser Elegie“ 1912. Er meint die Skala von Schönheit zur Hässlich- 
keit und als Endpunkt zum Schrecklichen, das ist die Domäne des erotisch fesselnden Eros, wie Rilke im Gedicht „Eros“ 1924 formuliert:

Masken, Masken! Dass man Eros blende.

Wer erträgt sein strahlendes Gesicht,

wenn er wie die Sommersonnenwende

frühlingliches Vorspiel unterstrich.

So kann es geschehen, dass solcher Anreiz überspringt von einem Menschen auf andere, die Spiegelneuronen [13] feiern Urstände, als wie vom Blitz geschlagen; „coup de foudre“ nennen es die französischen Romanciers trefflich. Ein Feuerwerk der Glückshormone (Endorphine) geschieht dem Empfänger, der überrumpelt, sich selber nicht mehr versteht. Masken sind im Spiel. Gesichter sind gemeint als Vorbilder einerseits und als Mahnung vor Missbrauch anderseits, vor dem Übermaß und damit dem Abgleiten ins Hässliche, ja ins Grässliche gar.

In der griechischen Mythologie wird die Liebesgöttin Aphrodite begleitet von Himeros, dem „Verlangen“, und von Eros, der „begehrlichen Liebe“. In der Tragödie „Antigone“ von Sophokles wird Eros vom Chor als unbesiegbar im Kampf angesprochen. Der Mensch sei der Macht des Eros, die ihn ergreift, gänzlich ausgeliefert. Das Dionysische, Animalische der menschlichen Triebnatur ist angesprochen. Eros wird in Ritualen eingefangen, religiös gezügelt, in Konventionen kultiviert und durch Gesetze geregelt. In der Neuzeit wird er neu beschrieben, als Freiheit verkündet und von Sigmund Freud als ein wesentlicher Grundtrieb des Menschen postuliert.

Also spielt Eros auf der genannten Skala unergründlich, überraschend, erfinderisch und unentwegt. Und die Menschen spielen mit, neuerdings besonders intensiv, und die Kosmetik bietet dazu Hand mit Ideen und mit Material.

Da sind die Clowns, auf der Skala auf halbem Weg, aber weg vom Schönen zum Komischen ( $\bullet$ Abb.3). Dem Gesicht wird mit weißem Aufstrich (gelegentlich auch als Maske) jegliche Struktur und Mimik genommen, die Augenhorizontale wird auf die zwei Augenhöhlen reduziert und nur der Mund wird enorm hervorgehoben und betont. Dies geschieht farblich durch Überzeichnung der natürlichen Lippenröte, durch Vergrößerung derselben und auch durch seitliche Ausdehnung der Mundpartie. Das Exempel Clown zeigt in Abb.3, welche Gesichtsanteile gelöscht werden müssen, um die komische Indifferenzwirkung zu erzielen. Im Umkehrschluss aber wird klar, dass ein differenziertes Gesicht mit Mimik und annähernd natürlicher Farbtönung, eine kräftige Augenhorizontale und der Blickpunkt Mund wichtig und nötig sind, um die besondere erotische Attraktivität zu erreichen. Diese kann allenfalls noch gesteigert, oder aber bis zur Hässlichkeit überzogen werden.

Am Beispiel Clown ist ersichtlich, dass eine Betonung der Mundpartie nicht in jedem Fall zur erwünschten Steigerung der Attraktivität führt, sondern kippen kann zum Komischen einerseits, oder dann zur Hässlichkeit bis zum Schrecklichen als extreme Kulmination. Dies gilt es, nochmals zu differenzieren. Mund und Mundpartie sind zentral im menschlichen Gesicht platziert. Sie stellen somit auch den zentralen Blickpunkt dar bei der Gesichtserkennung und den damit verbundenen Emotionen. Da es sich zugleich um eine starke erogene Zone handelt, ist es verständlich, dass sich um und durch Mund und Mundpartie besonders prononcierte erotische Affektionen drehen und entwickeln. Das Verlangen (Himeros) wird erweckt und Eros drängt zur Tat, dem Kuss. Viel geschehen, oft beschrieben, kann man Sophokles („Eros ist unbesiegbar“) verstehen und Sigmund Freud ebenso. Es liegt auf der Hand, dass die freizügige Kosmetik dem Verlangen

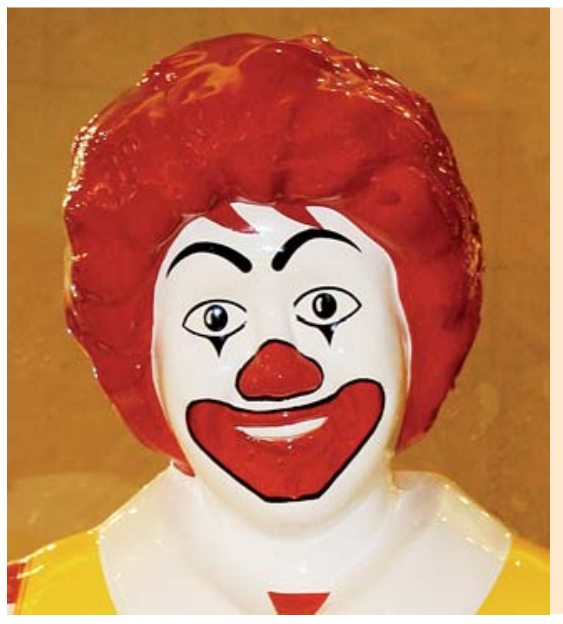

Abb.3 Der Clown Ronald McDonald. Der komische Ausdruck wird erreicht durch Reduzierung der Augenhorizontalen auf die zwei Augen, durch eine weiße Gesichtabdeckung und die rote Überzeichnung der Lippen. Komisch als ein gewisser Gegensatz zu schön (Bild: Chris Brown/zoonabar, www.flickr.com).

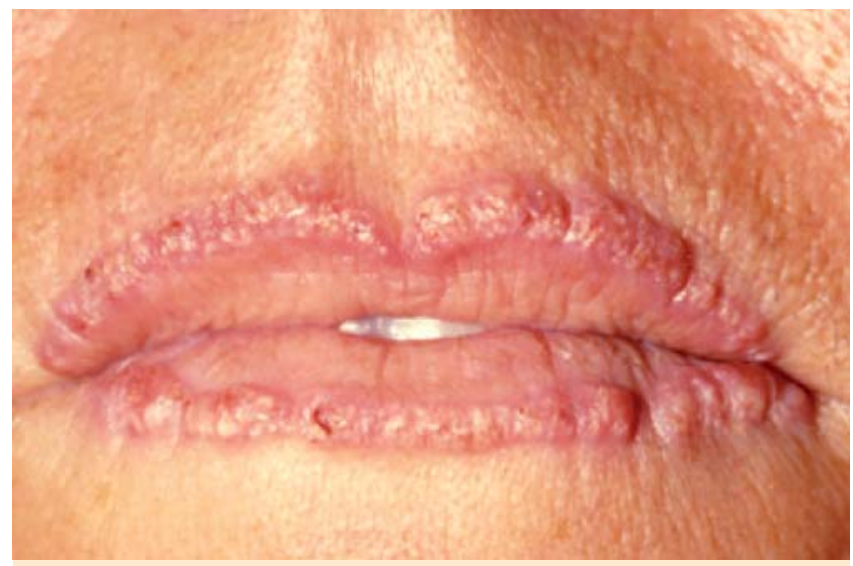

Abb.4 Markierung und Betonung des Lippenrandes als permanentes Make-up mit nachfolgender granulomatöser Überreaktion, eine schwerwiegende Nebenwirkung (Bild: Univ.-Hautklinik Mannheim, Prof. C. Bayerl und Dr. G. Feller-Heppt).

nach Steigerung dieser vorgerichteten Abläufe durch entsprechende Modifizierung der Mundpartie in vielfältiger Weise nachzukommen bemüht ist. Die betrifft sowohl die Farbe als auch die Größe und die Form, oder besser die Verformung.

Betrachten wir zunächst die Farbe: Das natürliche Lippenrot hebt sich bei heller Hautfarbe deutlich vom übrigen Gesicht ab, es wird durch Emotion und durch Druck infolge Mehrdurchblutung vorübergehend verdeutlicht. Eine Betonung des Lippenrots durch Auftragen eines roten Lippenstifts ist allgemeine Praxis und wirkt wohl auch zuverlässig. Zudem kann das Lippenrot durch Überzeichnung vergrößert dargestellt und durch einen Farbstrich nachgezeichnet und besonders kontrastiert werden. Letzteres geschieht durch Farbtätowierung, also Einbringen von Farbpartikel in und unter die Haut, ein permanentes Make-up. Die Persistenz ist gesucht, kann aber auch zum Nachteil werden, besonders dann, wenn ein Wechsel der optischen Wirkung angestrebt wird. Und dann birgt solche Tätowierung immer auch die Gefahr von unerwünschter Wirkung, wie Granulombildung ( Abb.4), Entzündung und Infektion [16-18]. Das permanente Make-up erwirkt nicht Attraktivität, sondern misswirkt bis zu Abscheu und Mitleid.

Die Erfahrung zeigt, dass absichtsorientierte Effekte durch fast alle Arten von Betonung des Lippenrots oder der Lippenfärbung als Kontrast zur Gesichtsfarbe erreicht werden. Farbe zeigt Wirkung, schafft beliebte „Hingucker“ und ist auswechselbar. Aller- 
dings sind die Kontraste abzustimmen auf die Hautfarbe, was bei dunkler bis schwarzer Haut zu sehr interessanten Lösungen führen kann. Dementsprechend bietet die Kosmetikindustrie praktisch alle Farbvarianten an ( $\mathbf{A b b}$.5) und lädt mit lockender Werbung zum Probieren und Anwenden ein. Mit der Farbe kann vieles versucht und manche der erwünschten Effekte können auch realisiert werden ( $\bullet$ Abb.6). Negative oder kontrapunktische Effekte sind nicht zu befürchten, außer bei den Tätowierungen.

Betrachten wir als Nächstes die Form des Mundes und die Gestaltung der Mundpartie. Hier gibt es deutliche Varianten des natürlichen Vorkommens vom „runden Spitzmäulchen“ bis zur breiten „Schublade“ oder „Suppenschlitz“, mit oder ohne sichtbarem Zahnsatz. Eine zusätzliche Verkleinerung wirkt eher niedlich bis lächerlich, ist also nicht erstrebenswert. Eine deutliche Verbreiterung führt zur seitlichen Aufrundung der Mundwinkel und birgt die Gefahr eines „Durchhängens“ der Lippenmitte, was dem Gesicht einen tristen oder abstoßenden Eindruck gibt. Dies aber sind Elemente, die einem Gesicht Hässlichkeit und „Schrecken der Medusa“ [6] vermitteln oder es in die Nische von Fastnachtsmasken [7] bringen. In diesen Bereichen droht operative oder dekorative Kosmetik unmittelbar in die Falle der Hässlichkeit zu führen. Überzeichnungen oder asymmetrische Weitungen des Mundbereiches, wie beim Clown ( Abb.3), führen ins Komische, und ein übergroßer, runder Mund zeichnet Krankheiten aus ( Abb.7) und weckt allenfalls Mitleid. All dies aber sind keine Elemente der sexuellen Attraktion, vom Eros nicht getragen und also außerhalb jeder kosmetischen Intention.

Die Nase wird in ihrer Struktur von Abweichungen befreit oder begradigt, und die Augenhorizontale erfährt vielfältige Variation des Haarbesatzes (Wimpern, Brauen), mit oder ohne farbliche Zusätze. Das Sprechende aber sind die Augen selber, die kaum verändert werden. Aber Bullaugen und Schielaugen sind krankhafter Natur, die behandelt, nicht kosmetisch angegangen werden sollen. Schlitzaugen und Lidstörungen hingegen werden zur Angleichung an besondere Ideale operiert, in der Hoffnung, zugleich Altersveränderungen zu beheben und Attraktivität zu steigern.

\section{Eros, Vater der Kosmetik \\ $\nabla$}

Inmitten der Erkennung von Gesichtern und dadurch ausgelöst, steht unbestritten die Wirkung auf den Betrachter. Empfinden, Abweisung oder Verlangen werden in kürzester Zeit geweckt. Haltung, Zu- oder Abneigung, verlangende Aktion, ja sogar Aggression sind Antworten. Locken und Reagieren aber durchziehen die Kulturgeschichte der Haut seit jeher und vielfältig [19

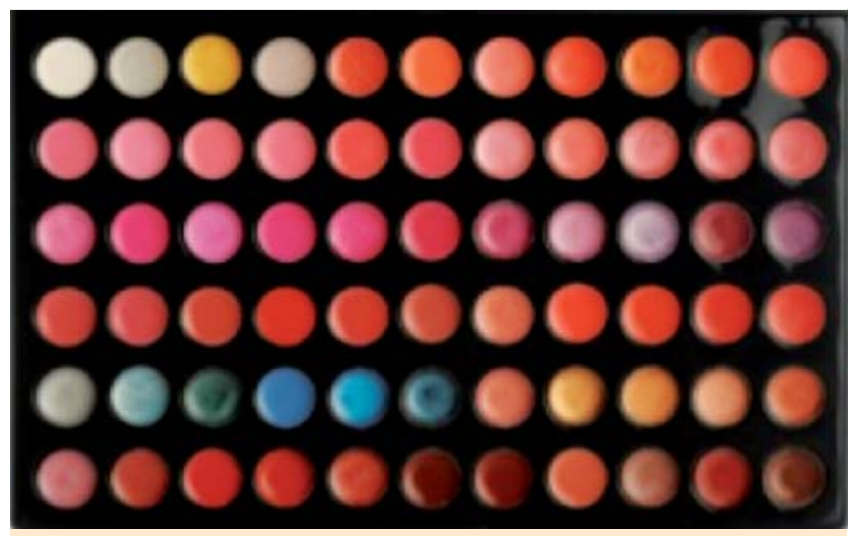

Abb.5 66 Farben kommerzieller Farbpaletten von Lippenstiften.

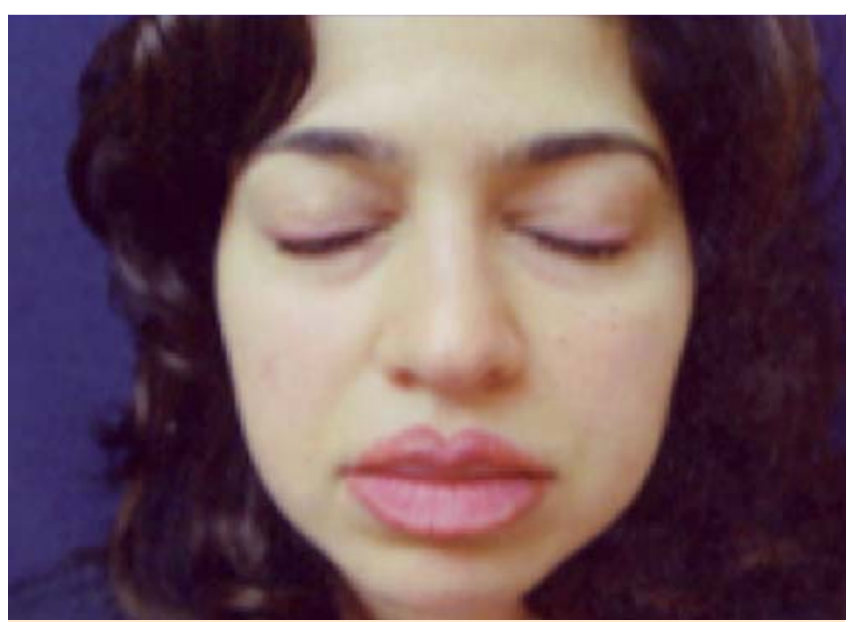

Abb. 6 Überzeichnung des Lippenrots mit starker Betonung der geschwungenen Form, verschiedene Farben (Bild: Prof. C. Bayerl, Wiesbaden).

-21]. Sie sind nicht wegzudenken, ebenso wenig wie deren Modifizierung durch die Kosmetik. Schönheit steht im Zentrum und erwirkt Wohlgefallen. Eros aber, der allgegenwärtige und unbesiegbare, verwandelt das Verlangen in „begehrliche Liebe“ und ruft zur Tat. Die gefällige Schönheit wandelt sich unvermittelt zur erotischen Attraktion. Die dazu bedeutsamen Elemente sind die Übersteigerung der Augenhorizontalen und vor allem die Betonung von Form und Farbe der Mundpartie. Dies ist das Feld der modernen Kosmetik mit all ihren Erfolgen und Fallstricken. Beispiele belegen dies. In $\bullet$ Tab. 1 wird versucht, die

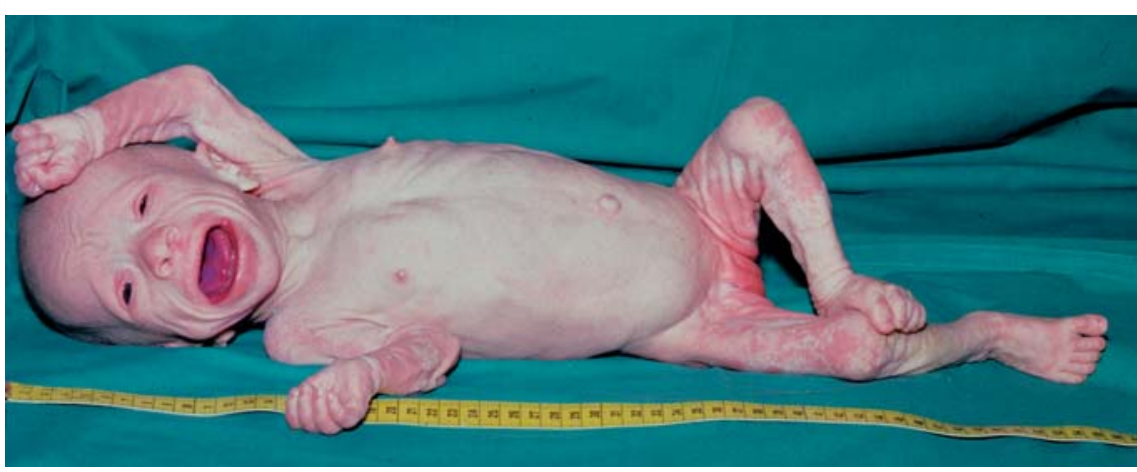

Abb. 7 Harlekin-Baby bei Ichthyosis congenita gravis, mit übergroßer, rundovaler Mundöffnung und angeborener generalisierter hyperkeratotischen Ichthyose des ganzen Integumentes. 
Tab.1 Darstellungsversuch der Beziehungen von Schönheit und Hässlichkeit mit deren Abstufungen und Übergängen.

\begin{tabular}{|ll|}
\hline Ausdruck und Wirkung & Symbole oder Exempel \\
\hline Hässlichkeit & Erkrankungen \\
\hline Mitleid & Krankheit und Entstellungen \\
\hline Komik & Clowns \\
\hline Schönheit & Ideal \\
\hline Erotische Attraktion & Eros gewinnt! \\
\hline Hässlichkeit & Masken \\
\hline Schrecklichkeit & Medusa \\
\hline
\end{tabular}

verschiedenen Formen von Ausdruck und Wirkung miteinander in Beziehung zu setzen und die besondere Relation der erotischen Attraktion zur Schönheit hervorzuheben.

\section{Interessenkonflikt}

Der Autor gibt an, dass kein Interessenkonflikt besteht.

$$
\text { Abstract }
$$

\section{Mythology and Cosmetics}

Cosmetics are based on mythology and are driven by Eros, the god of "desirous love". Eros is omnipresent in our consciousness as well as in unconsciousness; he operates in signs, emblems and symbols, and lives in rites and conventions und he waves deeply through our cultural development.

The recognition of human faces is the base of any reaction, moving between beauty and ugliness. Cosmetics tend to increase sexual attraction by modification of the faces elements. An over expression of the eye region as well as the modification in form and colour of the oral compartment are the preferred tools. Misuse may suddenly turn to ugliness, as can be demonstrated by mythological masks. This should be avoided.

\section{Literatur}

1 Assmann J, Hölscher T. Kultur und Gedächtnis. Frankfurt a. M.: Suhrkamp; 1988

2 Jung CG. Der Mensch und seine Symbole. London: Altus Books; 1964

3 Dawkins R. The Selfish Gene. Oxford: Oxford University Press; 1976

4 Rosenkranz K. Die Ästhetik des Hässlichen. Königsberg: Bornträger; 1853

5 Eco U. Die Geschichte der Hässlichkeit. München: Hanser; 2007

6 Jung EG. Perseus, Medusa und die Darstellung der Hässlichkeit. Akt Dermatol 2010; 36: 488 -491

7 Jung EG. Masken, vom Mythos zur Zeitkultur. Akt Dermatol 2013; 39: 90-94

8 Kläger M. Phänomen Kinderzeichnungen. Baltmannsweiler: Burgbücherei Schneider; 1990

9 Kläger M. Krampus: die Bilderwelt des Willibald Lassenberger. Hohengehren: Schneider-Verlag; 1992

10 Rorschach H. Psychodiagnostik. Methodik und Ergebnisse eines wahrnehmungsdiagnostischen Experiments. (Deutenlassen von Zufallsformen). Bern: Bircher; 1921

11 Kuhn R. Maskendeutung im Rorschachversuch. Basel: Karger; 1954

12 Kuhn R. Phénoménologie du masque à travers le Test de Rorschach. Paris: Desclée de Brouwer; 1957 (nouvelle édition 1992)

13 Jung EG. Das Phänomen Blickdiagnostik. Akt Dermatol 2011; 37: 214 217

14 Eco U. Die Geschichte der Schönheit. München: Hanser; 2004

15 Wietig C, Williams S, Reuther T et al. Zum ästhetischen Wertewandel in Kultur und Kosmetik. Akt Dermatol 2005; 31: 38-41

16 Schumann T, Peitsch WK, Geraud C et al. Ultraviolet light tattoo complicated by granulomatous inflammation. J Am Acad Dermatol 2011; 65: 124-126

17 Bayerl C. Tattoo und Haut. Akt Dermatol 2013; 39: 205

18 Stirn A, Möller J. Psychologische und medizinische Aspekte von Tattoo und Piercing - ein Update. Akt Dermatol 2013; 39: 228 - 235

19 Jung EG. Kleine Kulturgeschichte der Haut. Darmstadt: Steinkopff; 2007

20 Jung EG. Haut und Kultur, auch ein Gang des Geistes. Akt Dermatol 2008; 34: $437-441$

21 Jung EG. Kulturgeschichte und Dermatologie. Akt Dermatol 2010; 36: $192-195$ 